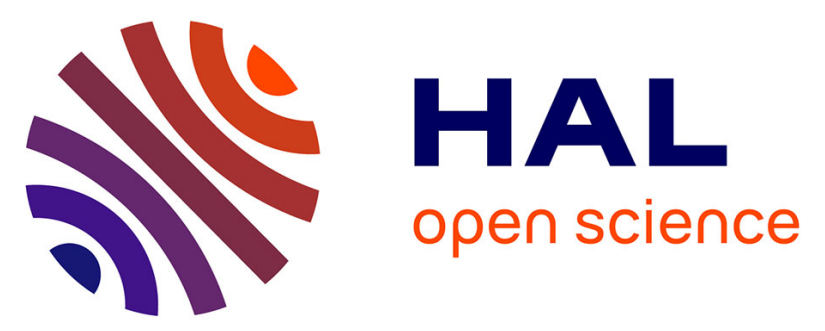

\title{
Les particularités du français calédonien (lexique, morphosyntaxe) et leurs enjeux sémantiques, pragmatiques et cognitifs
}

Didier Bottineau

\section{- To cite this version:}

Didier Bottineau. Les particularités du français calédonien (lexique, morphosyntaxe) et leurs enjeux sémantiques, pragmatiques et cognitifs. Langages, 2016, Le français calédonien (Nouvelle-Calédonie), une 'variété régionale' de français au sein de la francophonie. Confrontation de données calédoniennes, américaines, réunionnaises, mauriciennes, hexagonales, 203 (3), pp.49-69. 10.3917/lang.203.0049 . hal-01816877

\section{HAL Id: hal-01816877 \\ https://hal.science/hal-01816877}

Submitted on 15 Jun 2018

HAL is a multi-disciplinary open access archive for the deposit and dissemination of scientific research documents, whether they are published or not. The documents may come from teaching and research institutions in France or abroad, or from public or private research centers.
L'archive ouverte pluridisciplinaire HAL, est destinée au dépôt et à la diffusion de documents scientifiques de niveau recherche, publiés ou non, émanant des établissements d'enseignement et de recherche français ou étrangers, des laboratoires publics ou privés. 


\section{Les particularités du français calédonien (lexique, morphosyntaxe) et leurs enjeux sémantiques, pragmatiques et cognitifs}

\section{INTRODUCTION}

Le français parlé calédonien se caractérise par de multiples aspects tels que sa prononciation des nasales et des labiodentales, son lexique plus ou moins réservé aux initiés, la fréquence de certaines interjections. Ces marqueurs distinctifs constituent des clichés que l'on trouve mis en scène dans les blogs et certaines bandes dessinées comme La Brousse en folie (BF) de B. Berger :

(1) Lôngin ! et moi qu'allais barrer amener du bétail à Dédé ! T'aurais accouché toute seule... Mante dans la woiture, j'arrive! (Berger, La Brousse en folie, tome, 1985-2011)

Le français parlé calédonien présente également des singularités syntaxiques concernant l'adverbe (On a trouvé un nom plus que fin valabe...), la structure du groupe nominal (Petit bateau gros la cale), la négation (beaucoup laï 'pas du tout'), l'interrogation (Lôngin fifils, où que t'as mis la boîte de crayons de couleurs?). Ces traits ne sont pas suffisamment accusés pour faire totalement obstacle à l'intercompréhension avec le français zozo ('métropolitain') :

(2) Bonjour Monsieur! Ah ? Vous êtes Chinois ? Vietnamien ? Cambodgien? Parlez-vous le français ? - Comme toun'mande ! (source)

Mais la bande dessinée et les blogs peuvent faire montre d'un effort de rédaction dans un style «densément local » au point de rendre nécessaire une traduction ou la faire passer pour nécessaire :

(3) le cramou, il connaît fin bien se plônquer : le crabe mou sait l'art du camouflage. (source) 


\section{Le français calédonien (Nouvelle-Calédonie), une variété régionale de français}

Certains marqueurs lexicaux constituent une véritable marque de fabrique et sont repris pour dénommer des blogs, des radios, des programmes, comme Toutoute info sur Radio cocotier, la toutoute ou tututte étant un gros coquillage en forme de conque utilisé comme trompe dans la tradition kanak.

Le français calédonien doit en partie son originalité à l'hétérogénéité de ses sources lexicales et parfois grammaticales. On y trouve des emprunts et calques issus du gallo, du picard (faire montrer pour 'faire voir'), du breton (rester au sens de 'habiter' comme chom en breton), de l'anglais (creek, dungaree), de langues kanak, de divers créoles comme le bichelamar (cal. Boulouk 'costaud' / bich. Puluk 'taureau' ; on va kaïkaï 'manger') et on se rapproche parfois de phénomènes d'hybridation qui ne sont pas sans rappeler le tayo, créole de Saint-Louis (Pé a keu laï* 'il n'y en a plus du tout' (p. ex. de cigarettes), Pé a keu cinéma 'c'est raté pour le cinéma'), le drehu, kanak de Lifou (pëkö [pekø] 'zéro') et l'anglais (lie 'mensonge' grammaticalisé comme marqueur de la négation : beaucoup laï 'pas du tout', pé a keu laï forme une négation renforcée où sont grammaticalisés deux termes lexicaux issus de langues différentes, qui se cumulent sans se neutraliser).

Toutefois, il faut distinguer l'ethnolectal kanak, utilisé par des communautés dans leur cadre traditionnel, de l'ethnolectal européen qui se compose en négociant un mode de vie expatrié relativement à la métropole et une identité communautaire à travers des usages qui reflètent des habitudes et des routines de relations interpersonnelles.

Le français calédonien européen incorpore le lexique autochtone sous diverses conditions : le mot désigne une réalité locale (dawa 'poisson Naso unicornis'); le mot est porteur d'une interaction ordinaire, comme dire au revoir (tata!) ; enfin, le mot, par sa sonorité, évoque puissamment les propriétés empiriques de l'entité désignée et/ou permet divers jeux de mots : un requin (trapard) de bonne taille est qualifié de boulouk, qui n'est pas sans rappeler le français balaise / balèze du fait de la matrice consonantique consonantique $<\mathrm{b}-\mathrm{l}>$ en submorphémie lexicale (Bottineau 2014). Dans BF, le bébé Daisy est surnommée Kakoune ou Kakounette 'coup' (de poing ou de pied) ; cf. il lui a mis un kakoune :

(4) Héhéhé... en plus Daisy c'est juste un nom pour la mairie. Pour de vrai on va l'appeler « Kakoune » à cause des coups qu'elle donnait dans le ventre de sa mère... (Berger, La Brousse en folie, tome, 1985-2011)

(5) Mam ! Kakounette a fait caca! (Berger, La Brousse en folie, tome, 1985-2011)

avec un calembour évident, le mot cacounet étant aujourd'hui employé couramment sur les blogs en puériculture.

L'orchestration de ces composantes crée un ensemble linguistique cohérent révélateur d'une calédotitude, comme le fait dire B. Berger à l'un de ses personnages (amalgame de calédonitude et attitude, au sens parodique de "calédonienne attitude »). On étudie donc ici la diversité et la cohérence des ressources linguistiques endogènes et empruntées qui composent le français parlé calédonien tel 
que le met en scène B. Berger dans sa bande dessinée. Du côté de l'interprétation du système, on s'interroge sur la manière dont cette sélection de ressources, sa composition et son interprétation pragmatique contribuent à la production d'une " calédotitude linguistique » : un modèle commun de fonctionnement et d'interaction psychosociale, auquel participe l'individu par ses productions, à la fois en se conformant subjectivement à une mentalité normative partagée et en contribuant à faire émerger cette norme qui cristallise le groupe identitaire et l'effet de communauté. Cette problématique applique le paradigme de la linguistique enactive, qui définit la parole dans une langue comme un protocole incarné de synthèse du sens conforme à des pratiques normatives constitutives du groupe par l'interaction et le regard réflexivement porté sur elle. Certaines de ces constructions ne sont pas spécifiquement calédoniennes et se retrouvent dans nombre d'autres variétés du français, métropolitain ou non (nousautres, l'interrogation à double complémenteur). Mais, dans le cas du calédonien, le corpus Berger suggèrent que ces ressources, prises ensemble, forment un système de parole cohérent muni d'une coloration caractérisable.

Concernant le corpus utilisé, la bande dessinée n'est pas un corpus d'usage, mais une simulation concentrée, qui renforce le côté comique du personnage tout en caractérisant la " calédotitude » verbale de manière consistante. Il serait illégitime d'en tirer des mesures de fréquences. En revanche, vérification faite auprès de plusieurs informateurs, cette simulation dessine des scènes qui, à la lecture, se jouent réellement comme authentiques avec intonation et gestes imaginables : les constructions employées sont conformes à des usages effectivement vécus. Cette bande dessinée concentre une anthologie des usages effectifs, renforçant l'effet d'exotisme censé ressentir le « Zoreilles » et que s'approprie ludiquement le locuteur calédonien se mettant en scène dans et par sa propre parlure. La surenchère fait partie du jeu, mais souligne et caractérise d'autant mieux la dynamique des interactions spontanées.

\section{LEXIQUE}

Le lexique du français métropolitain constitue la ressource principale, mais il est diversement détourné de ses conditions et valeurs d'emploi conventionnelles. La première motivation est l'ajustement du lexique à la désignation d'un mode de vie dans un environnement particulier : le lexique français est réinvesti dans la désignation de mets culinaires, de plantes et animaux, de formations géographiques. Une porcelaine est une variété de langouste ; on baigne le bétail dans une piscine ; la gratte est une intoxication alimentaire causée par un aliment gratteux ; on chasse les gadins (cerfs). Le détournement passe souvent par la formation d'un composé, comme langouste porcelaine, où le premier terme thématise l'objet désigné et où le second rhématise une notion correspondant à une impression visuelle ou autre laissée à l'observateur : variété de langouste qui nous apparaît comme de la porcelaine; autres exemples : gateau huit, gateau trou, loup-maillon (jeu de poursuite), minerai chocolat ('nickel avec cassure fine' (Darot 1998) ; autres 
“04_Bottineau_2”(Col. : RevueLangages) — 2016/7/2 — 12:56 — page 4 — \#4

\section{Le français calédonien (Nouvelle-Calédonie), une variété régionale de français}

variétés : minerai écaille de tortue / moutarde / pain d'épices), merle moluques, pain marmite, poisson ballon, poisson ballon à piquants, tabac bâton. L'omission du terme initial est aléatoire : crabe carton qualifie l'état intermédiaire de l'animal entre la mue (crabe mou) et la carapace reconstituée, mais on ne parle apparemment pas de carton tout court en ce sens.

La construction est munie de sa narrativité : l'enchaînement des termes correspond à un parcours de découverte, la transition d'un élément générique connu (langouste) vers un élément spécifique inconnu (langouste porcelaine) identifié par une propriété sensible remarquable (porcelaine) d'ordre visuel ou autre, y compris du côté de la manière de préparer ou utiliser l'objet (pain marmite). Par suite, l'omission du terme de départ (une porcelaine) correspond à la normalisation de la propriété remarquable comme désignateur métaphorique de l'objet visé. Le procédé n'a rien d'inhabituel (français métropolitain : un sac banane, une banane), mais il est ici particulièrement répandu et, pour un observateur extérieur métropolitain, il produit deux impressions : d'une part, qu'une partie du lexique est très «empirique » et symptomatique au sens où il dénote l'impression sensible laissée par l'objet plutôt que l'objet lui-même : bouteille carrée pour 'bouteille de whisky', crocher pour 'attraper le poisson à la pêche', Chaleur ! pour 'le ressenti d'une émotion forte' (différent de Chaud ! en français, qui qualifie une excitation sexuelle, mais comparable au plus récent chaud bouillant 'difficile' ou 'dangereux'), Mouille! pour 'fais un effort' ; les gendarmes sont tendus (tôndus) après les infractions ou les jeunes femmes après les hommes qui leur plaisent. On emporte un pochon de mônger (pochon 'sachet', français métropolitain du grand ouest, ici complété par l'action programmée ; panneau d'une salle de l'Université de Lille 3 dans les années 80 : on peut apporter son manger) : on ne parle pas de la nourriture mais du rapport sensorimoteur entretenu avec elle. Une partie du lexique focalise le rapport aux choses plutôt que les choses elles-mêmes. Ce phénomène est ordinaire dans les parlers ancrés dans un mode de vie rural, où le lexique tend à encoder le ressenti de l'expérience matérielle, notamment par les étymons de P. Guiraud (1967), bien plus représentés dans les mots du quotidien agricole que dans une langue plus urbaine et intellectuelle, parlée par les citoyens d'un monde qui s'immatérialise. En psychologie enactive, la perception visuelle elle-même compose les objets en entrelaçant le traitement du signal optique avec la récupérations de simulations motrices mémorisées pertinentes qui font de l'objet visuel un prédicat muni de propriétés pragmatiques prévisionnelles, les affordances («faisabilités »), qui préparent le réel visuel à l'engagement du sujet agentif en le présentant comme parcourable, manipulable et prévisible.

Quand le lexique d'une communauté relatif à un Umwelt particulier reconduit cette dynamique, il tend à formaliser l'incarnation d'une culture par la communauté initiée : dans les composés, l'omission du terme de départ n'est possible que pour les initiés, et si les deux termes sont donnés, différentes manières de marquer la relation signalent différents niveaux de présupposition sémantique par le figement et d'appartenance au club pour les usagers. Outre l'omission du 
“04_Bottineau_2” (Col. : RevueLangages) - 2016/7/2 — 12:56 — page 5 — \#5

Particularités du français calédonien et enjeux sémantiques, pragmatiques et cognitifs

nom initial, on observe celle de la préposition (chien de bétail, chien bétail ; chou de chine blanc, chou chine blanc / vert, chou chine vert pomme). On trouve occasionnellement des constructions atypiques telles que poisson la mer ('poisson qui vient de la mer') ou Petit bateau, (mais) gros la cale ('c'est un petit bateau, mais elle est grosse, la cale', expression signifiant 'il ne faut pas se fier aux apparences' ; titre $\mathrm{d}^{\prime}$ un des albums de BF). Le tour a une dimension elliptique et ressemble superficiellement à des coordinations du français parlé comme petit cul (mais) gros cerveau et à des extrapositions adjectivales comme pas folle, la guêpe. Toutefois, d'un point de vue typologique, ce modèle rappelle aussi diverses composantes de la syntaxe de la langue bretonne, où l'on trouve :

- des focalisations en début d'énoncé (tomm eo an amzer 'chaud est le temps') ;

- des sujets disloqués (Per a zo brav e ti 'Pierre est beau sa maison' = 'Pierre a une belle maison') : en breton, on appelle « sujet disloqué » un sujet formulé en deux temps, typiquement le possesseur en position initiale préverbale, puis le possédé ou sujet « réel » en position post-verbale qui semble venir formuler une correction improvisée du déjà dit en amont, ce que signale l'absence d'accord en genre entre l'adjectif et le nom postposé, comme dans gros la cale en calédonien. Ce phénomène s'explique par une dynamique « chronosyntaxique » celtique où les syntagmes ultérieurs rectifient à l'interprétation les traits syntaxiques des syntagmes antérieurs (genre, nombre, fonction) sans que ceci n'apparaissent morphologiquement, d'où des effets de désaccord et, parfois, de double fonction (Bottineau 2012a).

Ce type de syntaxe est motivé par l'oralité de l'interaction, où se joue l'amorçage de l'énoncé par l'élément qui affecte le plus directement l'allocutaire, comme dans l'exclamation désho, ton crâne ('ta tête est déshonorée'), cf. en français lillois grillé, ta tête ('tu es vexé par ce que je viens de te dire, et c'est bien fait pour toi'). Divers travaux en ethnopsychiatrie (Carrer et al. 1983) ont montré que ces structures, qui contraignent le locuteur à choisir l'amorce de son énoncé en fonction de la relation attendue avec l'allocutaire, créent un entraînement psychosocial qui habitue le sujet parlant à déterminer par la parole ses états mentaux en fonction du rapport à l'autre beaucoup plus fortement que ne le font des langues comme le français ou l'anglais, dont la syntaxe est sous-tendue par la rhétorique de l'écrit (thématisations) plus que par l'interactivité dialogale. Le parler calédonien nettement est marqué par cette tendance.

La créativité lexicale et phraséologique prend parfois des proportions surprenantes. Ce qu'un Français métropolitain désignerait par la prison de Nouméa est dénommé tour à tour Camp Est, Les Six Quartiers, L'Île de l'oubli, La solitude, après le pont, la petite Australie, Los Angeles, le far west, Hotel California, stop-vacances, Bon-Courage, avec diverses motivations historiques, connotations et inscriptions ethnolexicales. On trouve dans $B F$ des énoncés où l'expression tronquée joue sur le double sens littéral et codé :

(6) avec ça y a moyen d'éclairer jusqu'en Australie. [ $\rightarrow$ l'île-prison, mais laquelle ?] (source) 
“04_Bottineau_2”(Col. : RevueLangages) — 2016/7/2 — 12:56 — page 6 — \#6

\section{Le français calédonien (Nouvelle-Calédonie), une variété régionale de français}

Le recyclage du lexique métropolitain prend un relief particulier dans certains champs sémantiques caractérisant le mode de vie. Dans le domaine de l'agriculture, certains collectifs comme bétail peuvent être dénombrés (des bétails). Dans le domaine des relations interpersonnelles, on ne compte pas les unités lexicales signifiant 'frapper' (panser, bourrer, astiquer, bombarder, damer, décalquer la gueule). De ce fait, l'intercompréhension entre français parlé métropolitain et calédonien fonctionne parfois beaucoup moins bien que ce que laisse attendre la proportion de lexique métropolitain apparent dans le discours : c'est que son recyclage crée un effet de code avec des relations signifiant/signifié reconditionnées de manière peu prévisible pour le non-local et souvent humoristique (chou fleur 'anémone de mer', perroquet banane 'espèce de poisson'), à la manière de certains textes de L. Carroll. La diversité des constructions est génératrice de polysémie : envoyer une boulette 'dire une vacherie', envoyer la boulette 'mettre les gaz', avoir la boulette 'avoir la forme', mettre la boulette 'faire l'action avec énergie' (cf. également, pour une voiture, avoir la chiasse 'puissance'). La polysémie peut être renforcée par les conversions de noms en verbe : boucan 'maléfice', emboucaner 'empoisonner' vs. boucaner 'sentir mauvais' et, plus généralement, la grammaticalisation : caillou 'bel homme' ou 'belle femme', mais aussi une marque de négation : ben caillou! 'ben non, pas du tout'.

Le recyclage du français métropolitain se manifeste également par l'application de certains procédés lexicogénétiques courants à du matériau inhabituel, comme la troncation : tal toul! 'à tout à l'heure tout le monde !', ceb 'c'est bien', d'où cebdemtal 'c'est bon, à demain ou à tout à l'heure', qui encode et condense tout un discours.

\section{COMMENT LE LEXIQUE DÉLIMITE UN MODE DE « VIE PARLANTE " ?}

Le parler français calédonien est utilisé par des groupes qui revendiquent explicitement leur conscience et leur plaisir et fierté de former un groupe caractérisé par un mode de vie, un mode d'expression et un mode d'interaction verbale modelés par une normativité psychosociale particulière. Cela se manifeste de manière cohérente à plusieurs niveaux.

\subsection{Désignation lexicale et pointage grammatical de la communauté}

Les Français métropolitains sont désignés de divers qualificatifs tels que les métro, les zoreilles / z'oreilles / zozos. L'origine de l'expression n'est pas certaine (Wissner 2016, ce volume) et on en trouve des remotivations populaires plus ou moins fantaisistes ; les deux plus courantes sont (i) le coup de soleil sur les oreilles qui distingue le nouvel arrivant à la peau très blanche, et (ii) la tendance des «zozos " à tendre l'oreille du fait de comprendre avec difficulté l'accent et le parler local. Par contraste, le groupe parlant le français calédonien s'autobaptise les nouzautes 'nous-autres', formation que l'on retrouve en espagnol 
normatif parlé et écrit (a nosotros nos gusta el turrón 'à nousautres nous plaît le nougat' $\rightarrow$ 'nous, nous aimons le nougat') et dans divers dialectes et français parlés régionaux, notamment le picard. Si le français parlé régional populaire spontané conserve nousautres dans de nombreuses régions, le français parlé se voulant normatif et l'écrit tendent à le proscrire, mais il s'est d'une certaine manière reconstruit sous la forme de l'opposition nous (borné, exclusif) vs. on (non borné, inclusif) : nous, nous aimons / on aime le nougat (le second nous réitère le bornage exclusif alors que on en détourne l'attention ; on n'existe pas comme pronom tonique du fait de son caractère non bornant, son inaptitude à focaliser l'attention sur une unité opposable à d'autres). Le chti possède un tizôt 'toiz-autres' correspondant à vouzôt en version tutoyée, que le parler calédonien n'utilise pas.

En français calédonien, la graphie de la construction en -autres varie continuellement, et avec une certaine cohérence : elle vise tantôt à souligner l'étymologie (graphie $a u$ ), tantôt à l'occulter (graphie o), renforcer le figement et souligner la prononciation (accent circonflexe, élision du $r$ ). Ces disparités correspondent à celles que l'on retrouve dans les étapes successives de la normativisation de l'orthographe du breton et du gallo, comme si la nécessité d'improviser la rédaction de l'oral confrontait les rédacteurs à l'ensemble des choix méthodologiques possibles que l'on retrouve pour la transformation des continuums dialectaux en langues.

En matière de distribution, les pronoms en -autres sont, comme en français québécois, indépendants et réservés à la position tonique et postprépositionnelle, en principe exclus des positions de clitiques sujet ou objet antéposés, avec quelques nuances toutefois : au Québec (Auger 1994), eux-autres est utilisable comme sujet lié dans le cadre d'une opposition (nous-autres on y va, eux-autres restent là); et de manière générale, nous autres complété d'un spécificateur admet cette position :

(7) Comme on peut le voir, nous autres Parisiens ne sommes pas si mal lotis que ça. (http:/ / www.timeout.fr ; consulté le 28-04-2015)

Cette construction en -autres est utilisée dès lors qu'il s'agit de présupposer l'existence d'un groupe constitué, avec affirmation identitaire, altérisation d'une minorité relativement à une majorité, et « appropriation individuelle d'un jugement générique » (Hilgert 2012). L'empan du groupe peut être étroit ou large :

(8) Ça y est le clebs ? Prêt à fouiller les gadins avec nouzautes ? (source)

L'emploi de sens large, qui réitère la revendication d'existence du groupe communautaire, permet de déclarer des oppositions et d'affirmer une identité distinctive. Dans les exemples infra, l'emploi de nous est possible et produirait l'effet de contraste, mais sans cette présupposition d'existence d'un groupe déjà établi avec une identité culturelle déterminée :

(9) Cete ané ya un jeune mètre fin valabe car il est un mec de la brousse come nousote les broussards (source) 


\section{Le français calédonien (Nouvelle-Calédonie), une variété régionale de français}

(10) Y a pas de voleurs chez nous-autes!

(11) [annonce dans le journal : Le « Club des Femmes Passionnantes » organise le concours des plus beaux inséparables] Un concours d'oiseaux ? Après ça, y en a qui croient que les femmes sont égaux! Mais nouzautes on a pas des idées comme ça ! (source)

Dans le corpus $B F$,-autres n'est pas utilisé si le clivage communautaire n'est pas présupposé (c'est moi qui souligne) :

(12) Z'inquiétez pas les gars, je vais faire montrer à ma mère que c'est la poupée de Kakounette qui joue avec nous ! C'est pas la Kakounette. (source)

(13) Eh ! quand tu vas rebarrer en Frônce les mecs vont être fin étonnés de savoir comment on crôche les crabes chez nous! (source)

[ces « mecs » ne sont pas censés avoir connaissance de l'existence de nouzaut en tant que minorité préétablie ; le point de vue adopté, allocentré, est celui de leur ignorance.]

Cf. aussi la transition nouzautes / nous dans (22) infra. Ce fonctionnement se retrouve à l'identique en picard, comme en (14) :

(14) Bonjour (tiote biloute), chez nouzaut's tout cha c'e des carabistoules! (site de l'association Nou'z'aut2 à Lezennes)

Nousautres intervient également dans l'expression de la possession. Le français calédonien alterne deux constructions possessives, comme le breton actuel, l'une antéposée, l'autre postposée :

(15) Et t'as vu la tête à toi ? (source)

(16) Hé ! tu connais, la trique elle est passée à ras les côtes à moi ! (source)

(17) Kevin le copain à moi (source)

Les composés en -autres se prêtent aux deux constructions :

(18) Hééé! M'sieur Nonold, vous faîtes pas le pont comme tous vôzôts collègues? (source)

collègues à vousôts serait ici mal venu : il soulignerait le fait que ces collègues ne sont pas ceux du locuteur, ce qui n'est pas pertinent dans ce contexte. Vos autres (possessif + adjectif) ne produit pas l'effet de clivage communautaire.

(19) la faillite à euzots (source)

Le caractère exclusif de -autres est mis en exergue par la position rhématique finale. Les deux constructions sont cumulables :

(20) Quand est-ce qu'elle termine leur grève des poubelles à euzots'? (source)

Le possessif antéposé, thématique, rappelle que « leur grève » est un présupposé connu de tous en contexte ; sa réitération en position rhématique par la forme exclusive en -autres permet de reposer l'opposition entre ceux qui font la grève et ceux qui la subissent, et souligne ce qui motive la question : non pas une demande $d$ 'information mais une exigence que prenne fin la discrimination entre les nouzôt qui subissons et les euzôt qui agissent. 
“04_Bottineau_2” (Col. : RevueLangages) — 2016/7/2 — 12:56 — page 9 — \#9

Particularités du français calédonien et enjeux sémantiques, pragmatiques et cognitifs

\subsection{Constructions avec verbes opérateurs}

Le français calédonien est riche en constructions à verbe support comme $<$ faire son $\mathrm{N}>$ (caicaisse, cacane, daguet, cowboy, coyote, jeulin....). Certaines restent proche $\mathrm{du}$ français métropolitain (faire son malin) et s'en distinguent par un lexique divers et coloré ; d'autres sont bien plus éloignées comme péter un coup de grève.

Une construction avec verbe opérateur permet de nominaliser une action reconnue comme typique et qui fait partie intégrante du répertoire d'activités constituant la norme culturelle du groupe : je vais chasser prédique ponctuellement une action d'un sujet sans souligner qu'elle relève d'un répertoire commun, tandis que je vais à la chasse fait du procès l'une des figures imposées du groupe auquel appartient le sujet et met en scène l'agent comme s'adonnant à une activité constitutive de son groupe culturel. Ce type de construction connaît un succès particulier en calédonien. D'une part, les activités sont quantifiées par le tour grammaticalisées un coup de (un coup de \{chasse, pêche, fête\}), qui explicite la dialectique de la généricité de l'action (sous article zéro) et de sa prise en charge par un vécu individuel (un coup est un événement). D’autre part, coup est précédé de divers verbes supports qui explicitent également la dynamique agentive de la prise en charge du procès (\{péter, claquer\} un coup de chasse) :

(21) Marcel : coup de chasse, coup de pêche, coup de fête et coup de gueule. (source)

(22) Voyez, la différence entre les zozos et nouzautes, c'est les sujets de conversation... Ouais !... nous, c'est totalemônt à l'opposé ! on parle de méga coups de chasse ! on est bien ! on parle de coups de pêche estraordinaires, de coups de fêtes canons! (source)

(23) J'te leur pète une bonne rafale aussi sec (source)

(24) Ha ben lôngin ! Pus peau de balle à la télé ! Woilà qu'y nous ont encore pété une grève ! (source)

Plus généralement, (se) péter et (se) claquer, sans un coup de, introduisent une action présentée comme initiée par un agent et dont la réalisation va produire un effet pour le groupe : Pète un café ! On se pète une bière / un apéro ? Dans le contexte du coup de chasse / de pêche, on peut péter ('prendre') une roussette / un poisson. Dans cet emploi, péter ne possède ni le sens vulgaire ni le sens de briser; il figure l'initiative individuelle qui affecte le groupe. On trouve à côté de cela des emplois plus proches des français européens tels que péter sa gueule pour 'se vanter' (cf. se la péter en français parlé actuel) sans rapport avec \{casser / péter / claquer\} la gueule de quelqu'un (violence physique, ou verbale pour claquer). La cohabitation d'emplois métropolitains avec les verbes supports calédoniens entraîne des divergences sémantiques quasiment énantiosémiques et manifeste des mécanismes d'érosion sémantique (bleaching) et de grammaticalisation comme le blocage apparent du pluriel : < des coups de $\mathrm{N}>$ est fréquent, mais pas après le verbe claquer (alors qu'en français québécois du discours sportif du football et de la course automobile, claquer des coups de \{buts / circuit $\}$ est très 


\section{Le français calédonien (Nouvelle-Calédonie), une variété régionale de français}

fréquent). La dimension culturelle et communautaire de l'expression coup de est soulignée dans $B F$ avec humour :

(25) Des machines partout et c'est l'humanité qui recule! Notre calédotitude qui se fait bouffer! Parce que, après les machines à laver, on aura les machines à coup d'pêche, les machines à coup d'chasse ! Les machines à coup d'plonge, les machines à coup d'fête et même, glups... les machines à coup d'gueule... (source)

\section{MARQUEURS DE SUBJECTIVITÉ}

Le corpus $B F$ fait apparaître que le parler français calédonien est particulièrement riche en marqueurs de subjectivité. On étudie ici leur cohérence et la manière dont leur synergie contribue à constituer un locuteur en tant que rôle social auquel se conforme le sujet parlant incarné et psychologique ; littéralement, un appareil discursif de l'énonciation.

\subsection{Interjections}

En premier lieu, il faut signaler le répertoire exceptionnel d'interjections et d'unités lexicales qui dénotent l'état émotionnel du sujet parlant ou, plus exactement, le moteur émotionnel de sa prise de parole.

\subsubsection{Interjections}

Interjections : awa, aouh, aouka, aoukaa

(26) Alors ? cette conserverie de bougna ?

- Awa ! ça vaut pas le coup... tous les mecs veulent faire pareil ! (source)

Cet $a w a$, probablement issu du phatique arabe $a w a$ 'ah bon', n'a rien à voir avec ben, euh ou bof. Il n'est pas traduisible en français, fonctionne comme pragmatème malgré son allure onomatopéique et, sans entraînement et pratique, on ne peut même pas se figurer précisément du modèle de ressenti émotionnel et psychosocial auquel il donne accès (cf. min ! en chti ou boh ! d'impuissance désappointée en italien). Le paradoxe des interjections dans les langues naturelles est qu'elles n'expriment pas diversement des modèles d'émotions communes, elles permettent au contraire d'apprendre à ressentir l'expérience de routines émotionnelles spécifiques, propres à des communautés, normatives du côté du signifiant et du "signifié » (si on accepte qu'un événement émotionnel en soit un), ancrées dans l'interaction. D'autres exemples pour se donner accès à ces ressentis :

(27) Awa les mecs ! faut qu'on ouve les yeux : nos conserves de bougna et ben caillou ! ça se vônd pas! (source)

\subsubsection{Noms interjectifs}

Noms interjectifs : Baptiste! Babylone! Microbe! Pistache! Kékouya! Bataillon! Bataillon Lafayette! Calice! Calisson! L'empété ! Lônculé ! Lôngin! 
“04_Bottineau_2” (Col. : RevueLangages) — 2016/7/2 — 12:56 — page 11 — \#11

Particularités du français calédonien et enjeux sémantiques, pragmatiques et cognitifs

Ce dernier (Lôngin !) correspond souvent à 'bon sang' en français, mais il s'emploie infiniment plus et pour toute la gamme des émotions allant de la surprise à l'exaspération ou la rage (l'é-motion est par définition le pic physiologique et ressenti qui se produit dans une situation inattendue, quelle que soit l'orientation de son recadrage en termes de plaisir, douleur, appréhension, colère, etc.). Lôngin est le marqueur emblématique et générique du motif émotionnel de la parole, et sa fréquence souligne que dans la routine psychosociale à laquelle le français calédonien conforme le sujet, la parole émue, ou l'émotivité parlante, est une expérience ordinaire.

(28) Il a bougé lôngin ! t'as pas senti ? [surprise] (source)

(29) Mais lôngin ça sert à quoi un bébé ? Kakounette passe presque tout son temps à dormir! [il n'y a pas d'exaspération ici, juste de l'étonnement] (source)

(30) Lôngin fifils, où que t'as mis la boîte de crayons de couleurs ? [impatience] (source)

(31) Lôngin de lôngin de lôngin de lôngin de lôngin ! jamais vu ça ! [culmination de la surprise et de l'exaspération] (source)

\subsubsection{Locutions et phrases interjectives}

[admiration, satisfaction] (fr. super, génial, extra, top) : ça de wizz, ça de wo, ça de yeah ! ça dose ! valable ! boulette ! kalolo ! tchi!

[rejet] ça de no $(z)$ ! en ethnolectal kanak : tcha!, tchia !, kia! (avec divers degrés d'allongement de la voyelle), qui peuvent être utilisés en réaction de désapprobation dans le dialogue ou en amorce énonciative d'un propos animé par une émotion négative.

Le français parlé actuel utilise moins de marqueurs comparables, mais on en voit se développer comme simulation écrite de l'oralité dans les blogs, comme pouarf, arf, niarf, pseudo-interjections simulées, initiales ou finales, qui permettent de jouer un motif émotionnel.

Tout se passe comme si l'amorçage de la prise de parole passait par l'affichage du moteur émotionnel qui la suscite, à la manière d'une réponse verbale à un stimulus non verbal. Ceci est tellement vrai que le lexique de la diction est luimême profondément affecté par le rapport à l'émotion. Le verbe parler entre dans nombre de constructions comme verbe support comme Parle bien! ('ne sois pas vulgaire'), parler mal 'médire', parler mauvais, avoir une/la bouche qui parle mauvais. Avec le verbe dire, on trouve Dis bien! (agacement ou rejet du propos d'un interlocuteur soupçonné d'avoir menti), Dis pas! (surprise 'ça alors !'), Dis pas fort! ('La honte!', 'oups!' dans son emploi actuel : évaluation de l'action d'autrui, verdict négatif).

Or, cette dimension émotionnelle n'est pas un facteur anecdotique, mais une variable typologique. Un cas extrême de ce genre est celui d'une langue du pays Dogon où les modes verbaux représentent l'esprit extérieur au sujet parlant qui inspire la parole selon qu'elle exprime une connaissance, un désir, une appréhension, une réaction de colère : le sujet parlant n'est pas prédonné comme unité centrale et agent source de sa parole, mais comme médiateur ou 


\section{Le français calédonien (Nouvelle-Calédonie), une variété régionale de français}

relais inspiré par des sources extérieures hétérogènes. En allemand parlé actuel, tous dialectes confondus, les productions des locuteurs contiennent un nombre important de marqueurs comme doch, ja, naja, nun ja, qui spécifient le type de réponse émotionnelle ou de réactivité dialogale qui préside à la prise de parole instantanée, et qui fait du dire un relais ou une médiation plutôt qu'une source (ce fait est souligné par certaines méthodes d'allemand comme Naja! qui font de telles interjections leur titre). Si l'on compare des bandes dessinées, on ne trouve aucune bande dessinée francophone qui contienne une telle diversité et densité d'interjections que $B F$, même dans des séries comme Titeuf de Zep, dont les personnages enfantins interagissent pourtant continuellement sur les modes émotionnels de l'enthousiasme, du conflit, de l'exaspération. Certaines traductions de Titeuf, en créole (Le sens de la vie : chimen lavi pour un mélange de créoles caribéens et guyanais et koulër la vi pour le créole réunionnais), en breton (red ar vuhez) et en allemand (der Sinn des Lebens) contiennent sensiblement ou nettement plus de marqueurs de ce type. En allemand en particulier, la comparaison de corpus de bandes dessinées traduites, sur des séries entières, livre des résultats sans ambiguïté : il n'y a jamais moins du double d'interjections en allemand par rapport au français, quel que soit le sens de la traduction. Dans cette langue, l'explicitation du moteur subjectif de la prise de parole fait partie intégrante de la vie énonciative, et ce paramètre différencie l'expérience de la parole dans cette langue par rapport au français ou à l'anglais.

Or, l'expression de ce moteur ne passe pas que par des interjections et connecteurs, il est également grammaticalisé. En anglais, la forme progressive est utilisée non pas pour mettre en durée le procès mais pour « mettre au ralenti » sa figuration interprétative et imposer à l'allocutaire le temps de détecter que le locuteur est mû par une pulsion liée à la mise en débat, voire la polémique : if we are not playing golf, I'm not going anywhere! 'si on ne va pas au golf, moi je ne vais nulle part!' (conflit conjugal entendu dans la rue ; en calédonien, on commencerait par lôngin !). En breton, l'expression d'une habitude passée se fait au mode dit indicatif si le locuteur est animé par l'extériorisation d'une connaissance personnelle formée à l'occasion de l'observation récurrente d'événements dont il a été le témoin, alors qu'elle se réalise au mode fréquentatif s'il s'agit de répéter une vision communément formulée par les membres de la communauté culturelle. L'inuktitut (langue eskimo : Groenland, île de Baffin) fait la même distinction et construit la négation différemment selon ces modes, sachant que nier en son nom personnel ou en celui du groupe n'est pas la même chose. En espagnol, en particulier sud-américain, le verbe ser 'être' est utilisé pour un jugement conventionnel auquel le locuteur se conforme alors que estar 'être' formule un jugement personnel assumé comme distinctif par rapport au jugement d'autrui : Sharapova es buena, y está buena 'Sharapova, c' est une bonne, et elle me plait'. Tous ces exemples concernent la manière dont le sujet parlant se voit imposer - par des moyens grammaticaux et lexicaux une habitude, voire une obligation - de se présenter comme relais énonciatif et de spécifier la source émotionnelle interne ou dialogique externe de sa prise de parole. Le français 
dispose de relativement moins de marqueurs de ce genre, mais le parler français calédonien, au vu des dictionnaires et des simulations d'usage fournies par la bande dessinée, renforce considérablement le dispositif par divers moyens.

\subsection{Reduplications}

Les reduplications sont comparables à celle de l'italien (piano-piano 'lent-lent' 'tout doucement') ou du basque (isil-isilik 'lent-lent' (partitif) 'tout doucement'). Certaines sont communes au français métropolitain comme pas sympa-sympa pour réprouver de la malveillance :

(32) Le calme plat pour la coupe du monde de kite-surf, c'était pas sympasympa... La pluie pour le concert du groupe anglais UB40, c'était pas sympasympa non plus... mais alors, le vent et la pluie pour le pont du 11 novembre, faut pas exagérer !!! (http:// croixdusud.info)

Certaines reduplications concernent un groupe adverbial :

(33) Je ne vous ai pas vu du tout du tout! (source)

voire le groupe verbal :

(34) Woila tu wois : en voici quelques unes. (source)

Ce modèle rappelle des expressions bretonnes courantes comme gouzout a ouzon 'savoir je sais' $\rightarrow$ 'je sais (bien)', que l'on entend parfois calquées en français parlé de Basse-Bretagne.

\subsection{L'adverbe fin}

Dans le domaine de la prédication, le marqueur de subjectivité le plus utilisé et grammaticalisé est l'adverbe fin :

(35) Un bateau de touristes australiens! Ce sont les commerçants qui vont être fin contents (source)

Certains de ses emplois sont traduisibles par tout, très, bien ou vraiment en français, mais fin est employé en calédonien avec une fréquence qui ne correspond pas au français très (utilisé pour la surenchère), bien (pour la confirmation) et vraiment (pour la certification de la véridiction). On le trouve en picard (fin biau 'très beau', fin bénache 'bien à l'aise', 'confortablement installé', fin héreux 'bien heureux'), souvent avec une appréciation positive ou négative ( $i$ a eu fin caud à sin tchoeur 'ça lui a fait chaud au cour' ; fin rosse 'bien abîmé' après une bagarre ; fin raite 'dense' (pour du charbon)). L'emploi calédonien a pu partir de cette valeur appréciative, qu'il conserve parfois, mais il s'est considérablement élargi. Il n'a pas non plus la duplicité des préfixes extra-, ultra-, archi-, super-, hyper-, méga- et giga- du français parlé actuel, où se joue un conflit ludique entre la surenchère émotionnelle, hyperbolique et transgressive (super méganul, trop gigagénial, de plus en plus mégachouettes, la plus méga sympa des mamans) avec l'emploi d'un paradigme de préfixes savants qui présupposent une classification érudite invoquée par un locuteur froid, conformiste, déguisé en expert ; ce mélange saugrenu est devenu conventionnel en parler jeune (on n'imagine pas comme mention sur 


\section{Le français calédonien (Nouvelle-Calédonie), une variété régionale de français}

une copie d'examen trop / super / méga bien... sauf à se jouer des conventions). Fin calédonien ne produit aucun de ces effets. Dans le passage de $B F$ où B. Berger s'amuse à traduire des passages calédoniens en français, il se garde bien de traduire l'adverbe :

(36) le cramou est fin malin : le crabe mou est rusé (source)

(37) il connaît fin bien se plônquer : il sait l'art du camouflage (source)

Du point de vue sémantique, fin, lié à fini, comme dans le français un con fini, permet de certifier que l'attribution de la propriété adjectivale ou autre est réalisée sans réserve et s'applique de manière complète. Dans le cadre guillaumien de la psychomécanique du langage, on dirait que l'incidence de l'adjectif au verbe est complète et que l'adverbe fin grammaticalise la notion d'achèvement en l'appliquant à l'opération métalinguistique d'attribution de propriété, la référence de l'apport au support. Dans le cadre culiolien de la théorie des opérations énonciatives et prédicatives, on traiterait la question en modélisant le parcours du domaine notionnel construit par le couplage du nom et de l'adjectif. L'effet de sens est que 'le cramou est complètement digne d'être qualifié de malin', ce qui revient à souligner le rôle du locuteur comme observateur réflexif de sa propre production sémantique : se posant en arbitre métalinguistique, il s'assure du contrôle des opérations de parole et certifie la conformité des mots au sens visé face à l'allocutaire. Le français réserve cette opération aux cas où le locuteur différencie sa position par surenchère (très malin) par rapport à la position de référence (malin tout court) que pourrait formuler tout locuteur non identifié : en disant le cramou est malin, le locuteur s'assimile à tout autre locuteur possible et réitère une doxa sans se distinguer ; avec fin malin, le locuteur réitère et vérifie la doxa, faisant de son opinion personnelle un acte d'expertise. En français calédonien, cet adverbe fin permet au locuteur d'affirmer son regard et son autorité même quand ce qu'il a à dire ne se distingue pas de ce qu'aurait dit tout autre à sa place. L'effet est paradoxal : d'un côté, il souligne la subjectivité « à vide ", comme dans ce titre d'un film de J. Yanne $C^{\prime}$ est pas parce qu'on n'a rien à dire qu'il faut fermer sa gueule; la formule est ici c'est pas parce qu'on n'a rien à dire de plus qu'il ne faut pas se montrer. D'un autre côté, fin souligne cette subjectivité de manière rituelle quasiment obligatoire ou réflexe, donc inintentionnelle ou inconsciente, conformant de fait la manifestation de subjectivité à un modèle psychosocial de l'interaction caractéristique de cette calédotitude. Cette forme illustre un point essentiel de la linguistique enactive : l'agent parlant individuel « incorpore » des modèles verbaux de codétermination psychosociale normative ; en se conformant à des protocoles de diction fournis comme modèle, il s'approprie et naturalise des routines culturelles ; ce faisant, il forme sa "subjectivité de surface ", celle des interactions verbales : il apprend des actes d'humeur et des ressentis à travers l'interaction routinière, intériorisant des modèles émotionnels partagés. Cette conception de l'émergence interactive du sujet " culturel », en fait psychosocial, par ses engagements incarnés avec protocole normatif, s'appuie sur la notion merleau-pontyenne d'engagement et son dépassement par la notion de coordination incarnée chez H. Maturana et 
“04_Bottineau_2”(Col. : RevueLangages) — 2016/7/2 — 12:56 — page 15 — \#15

Particularités du français calédonien et enjeux sémantiques, pragmatiques et cognitifs

dans le paradigme de la cognition dite « des quatre E » (embodied 'incarnée', embedded 'située', enactive 'enactive', extended 'étendue' technologiquement), qui rivalise aujourd'hui avec le paradigme cognitiviste ancien (représentationnaliste et calculatoire).

Ce fonctionnement de fin et son rôle psychosocial explique l'énorme succès du marqueur, quantitativement d'abord, mais aussi par la diversité de ses inscriptions syntaxiques. Fin commente l'adjectif :

(38) Je vais rômplacer mon taureau ! il est fin usé ! (source)

Vu que fin ne démarque pas le contenu du jugement de celui qu'aurait pu avoir un autre locuteur, on le trouve dans des structures polyphoniques, typiquement des subordonnées dont la source est attribuée à un énonciateur dissocié du locuteur, ce qui est impossible en français avec très, tout, vraiment :

(39) Bizarre... là haut y a que le vieux gadin que personne ne tire tellemônt qu'y doit être fin dur... (source)

Ces emplois corroborent la neutralité de fin: pour que l'intensification soit réelle, elle doit être renforcée par d'autres moyens. Pour la même raison, le fin calédonien est compatible avec tous les degrés de l'adjectif (comparatif, superlatif) :

On a trouvé un nom plus que fin valabe... (source)

(41) Kakounette, elle est de plus en plus fin jolie! (source)

Le français admet marginalement ce type de construction (c'est plus que très cher), mais seulement lorsqu'il s'agit d'une réplique à un interlocuteur. Si seul l'axe dialogique est impliqué, si le locuteur met en scène des énonciateurs fictifs qui ne servent qu'à adosser sa propre position, comme avec de plus en plus, l'adverbe est exclu en français ( ${ }^{*}$ de plus en plus très / tout / vraiment + Adj.). Avec le superlatif :

(42) la plus fin valabe des mamôns (source)

Dans ces trois derniers exemples où il est question de la mère et de la fille, le locuteur ne peut se contenter de fin s'il tient à souligner la sincérité de son jugement. Fin commente aussi l'adverbe :

(43) Je ferai des récrés qui durent fin langtemps! (source)

Puisque fin se borne à poser le locuteur comme contrôleur de l'adéquation intégrale de la prédication, rien ne s'oppose à ce qu'il porte sur l'adverbe bien :

(44) (un écologisse) c'est un mec qui aime fin bien la nature (source)

Avec sa polyvalence polyphonique, fin permet également de certifier une négation ; dans l'énoncé suivant, on croirait entendre un Titeuf nouméen :

\section{C'est fin pas juste (source)}

Comme on le constate, le calédonien présente une gamme d'opérateurs cohérents de producteurs interactifs de la subjectivité. Corollairement, cela suppose qu'il dispose également d'un appareil de la construction de l'autre. On aborde donc la question de l'allocutivité. 


\section{Le français calédonien (Nouvelle-Calédonie), une variété régionale de français}

\section{MARQUEURS D'ALLOCUTIVITÉ}

L'allocutivité réside dans l'utilisation de formes langagières permettant de coordonner les états de conscience subjectifs relativement à la relation soi/autrui en les amalgamant ou en les discriminant. L'enactivisme, à la suite de L. Vygotski et $\mathrm{M}$. Merleau-Ponty entre autres, considère que si les agents parlants que l'on observe apparaissent comme préétablis et robustes du fait de se manifester par des corps autonomes et discrets, du point de vue réflexif et intérieur, ils ne se discriminent pas continuellement comme des entités psychologiques séparées, mais le font au travers d'activités non verbales et verbales. En basque, on peut dire 'il fait beau' à la conjugaison «normale » eguraldia ona da ou à la conjugaison allocutive eguraldia ona duk/dun, laquelle porte un marqueur de deuxième personne tutoyée masculine $(k)$ ou féminine $(n)$ marquant la reconnaissance de l'autre dans ce qui le distingue de moi et le différencie de tout autre allocutaire possible : le basque grammaticalise la possibilité de constituer l'altérité intersubjective par la relation allocutive. En théorie des systèmes grammaticaux, cette problématique est l'un des enjeux de la Théorie de la Relation Interlocutive de C. Douay et D. Roulland (2014). Pour le calédonien, nous nous intéressons ici à des faits de discours et d'interaction plus qu'à l'auto-organisation du système de la langue, mais nos analyses vont dans la même direction. Les faits d'allocutivité que nous relevons concernent deux moments de l'interaction : sa mise en place par l'interpellation et sa gestion par la coordination des points de vue.

\subsection{Interpellations et insultes}

L'interpellation est assurée par une diversité remarquable de termes lexicaux : (copain, cousin, frère, mon con, ma couille, vieille douille, vieux). Certains sont français, d'autres de langues locales. Certains ont une origine très vulgaire, mais la connotation est neutralisée. Tout se passe comme si l'important était d'aborder l'autre en le faisant exister comme être singularisé par des moyens lexicaux choisis en guise de reconnaissance. Cela concerne également les insultes, termes $\mathrm{d}$ 'adressage et de désignation personnelle dépréciatifs, qui peuvent être très colorés (pine, tête de pine, pine d'ours) mais peuvent voir leur vulgarité neutralisée en contexte affectif ( $m a$ couille), comme con en français parlé (en particulier toulousain). Plus généralement, cette neutralisation débloque des emplois métaphoriques : (se) taper la pine peut signifier 'se masturber' ou 'se faire des illusions' (tu tapes la pine ou quoi?). Ces usages montrent à quel point la gestion de l'intersubjectivité l'emporte sur la désignation des entités. À la limite, tous les moyens lexicaux sont potentiellement bons, du moment que la situation et l'interaction (intonation, positions, gestes, regards, mimiques), dans sa dimension partagée et codifiée, orientent correctement les effets produits par les termes utilisés.

\subsection{Coordination intersubjective}

La coordination intersubjective régule la convergence des savoirs et points de vue et gère la dynamique des actes de concorde et de discorde. Elle est 
“04_Bottineau_2” (Col. : RevueLangages) — 2016/7/2 — 12:56 — page 17 — \#17

Particularités du français calédonien et enjeux sémantiques, pragmatiques et cognitifs

réalisée par des formes de fin d'énoncé qui préparent la poursuite du dialogue en thématisant l'accord et l'intercompréhension, comme ou quoi :

(46) Oh, Dédé ! il est ban ou quoi ? [comment ça va ?] (source)

(47) Allô allô, il est ban ou quoi ? (source)

Comme avec l'adverbe fin, il s'agit d'une mise en scène routinière, rituelle, inconsciente et inintentionnelle de la relation interlocutive. Ce ou quoi ? n'est pas la réponse verbale à un stimulus émotionnel ressenti de type impatience ou exaspération (français il est dingue ou quoi ?), il est l'automatisme codique qui conditionne la poursuite du dialogue (à la manière de certaines question tags en anglais). L'allemand parlé actuel présente des emplois assez proches dans des contextes du type : Alles klar, oder..., Alles klar, oder was ? aux côtés d'emplois identiques à ceux du français : Spinnst du oder was ? / oder so ? 'tu déconnes ou quoi ?'. Une langue comme le basque permet en fin d'énoncé le choix entre un ou exclusif ala (erotuta haiz ala?), qui pose une alternative oui/non, un dilemme, et un ou inclusif edo (erotuta haiz edo ? 'tu es fou ou bien... (il se passe autre chose ?)'), qui associe l'allocutaire à la sélection des options possibles qu'il convient de poser en vis-à-vis de la déclaration de départ. En français populaire des jeunes en France, on trouve également un ou quoi ? très fréquent en fin de question qui ne signifie pas l'invective mais l'incitation à répondre, plus pressante qu'en calédonien.

Les marqueurs allocutifs qui préparent la réception de l'énoncé se trouvent également souvent en début de réplique, et le calédonien parlé en fait un usage abondant et diversifié : Hé ! ('fais attention à ce qui suit' ; différent du français hé ! qui réveille un allocutaire inattentif). Le succès de la réception est luimême entériné par divers marqueurs tels que ben alors! ben ça! ben c'est ça! qui soulignent la complicité des interlocuteurs, un peu comme en français tu m'étonnes! 'tu me surprends pas du tout', 'compte tenu de ce que nous savons, il fallait s'y attendre, et nous étions sur la même longueur $d^{\prime}$ onde'. Le tour $<c^{\prime} e s t$ bon (si) + phrase> formule une nécessité de manière euphémisée ('il faut que') et le terme moyen, grammaticalisé comme moien en breton, entre dans diverses structures négociant la possibilité : Moyen (bon)! 'c'est possible', Moyen (bon) (de) + infinitif (Moyen monter dans le car? Moyen faire ?). Le calédonien ponctue le dialogue de ce type d'opérateur d'accord interpersonnel, gérant en continu les phases de concorde et de discorde.

\subsection{L'interrogation avec relative}

Dans la série $B F$, les interrogations partielles avec pronom qui, que, où, quand, comment sont presque toutes renforcées par une relative. Cela n'est pas propre au français calédonien et apparaît dans divers parlers régionaux métropolitains, mais la construction prend un relief intéressant dans notre corpus et alterne avec l'interrogation simple (47) :

$$
\text { Qui qui veut du thé ? (source) }
$$


“04_Bottineau_2”(Col. : RevueLangages) — 2016/7/2 — 12:56 — page 18 — \#18

\section{Le français calédonien (Nouvelle-Calédonie), une variété régionale de français}

(49) Mon réveil ! qui qu'a pris mon réveil ? tu vas voir la pônse à celui qu'a pris mon réveil !!! (source)

(50) Lôngin fifils, où que t'as mis la boîte de crayons de couleurs ? (source)

(51) Awa dis pas Tathan!? Depuis quônd que t'as un clébard ? [avec awa de surprise et dis pas de réfutation du propos correspondant à la vision qui suscite l'incrédulité] (source)

(52) Aouh ! Marcel où tu barres comme ça ? (source)

Avec la relative, l'allocutaire visé par la question est présenté comme détenteur d'un savoir ou d'un projet d'action qu'il s'est déjà formulé et qui peut se gloser sous la forme d'un dire : Qui qui veut du thé ? 'qui a déjà fait son choix de boire du thé ?'. Dans $B F$, Marcel emploie la relative à chaque fois qu'il prête à l'autre une intention cachée : qui qu'a pris / où que t'as mis mon (réveil, manteau...). Son épouse, quant à elle, surprise par son départ, lui demande l'information innocemment, sans lui prêter ce type d'intention cachée (où tu barres ?) alors qu'elle pourrait le faire (où que tu barres?). L'interrogation avec relative permet une mise en scène polyphonique : la personne interrogée est figurée par deux instances, celle de l'allocutaire en interaction dialogale immédiate avec le locuteur et celle d'un énonciateur préconstruit compris comme source d'un dire présupposé et antérieur à l'acte $\mathrm{d}^{\prime}$ interrogation. Cette mise en scène est une routine : elle ne correspond pas nécessairement à une réalité psychologique du côté de l'interrogé ni à une intention de mise en scène du côté de l'interrogateur ; mais, en tant qu'habitude de présentation, elle structure les rôles conventionnels distribués dans l'interaction, et c'est sa suspension occasionnelle qui se remarque le plus. Une interrogation réflexive avec se demander permet également cette mise en scène :

(53) De fois, je m'demônde où qu'il va chercher toutes ses histoires ! (source)

De la même manière, on distingue je me demande si et je pense que si, qui « polyphonise » le locuteur en tant qu'allocutaire réflexif visé par la question et présenté comme détenteur préalable de la réponse qu'il s'agit de faire ressortir :

(54) - Hé ! Longin tu pointes ou tu tires?

- Dérôngez pas ! Je pônse ! Hé ! Faut jouer avec la tête aussi !

- Tu pônses à quoi ?

- Je pônse que si je pointe un coup ou si je tire un coup... je pônse quoi ! (source)

La polyphonisation est quasiment de règle avec l'emploi du verbe connaître en lieu et place de savoir :

(55) Tu connais où que Dédé et son mari y zont été pêcher ? (source)

En français, savoir introduit une prédication ponctuelle, qui peut être développée (je sais que deux et deux font quatre) ou condensée et anaphorisée (je sais cela) ; connaître introduit un domaine de connaissance dont le contenu résulte de la cumulation d'un ensemble ouvert de prédications (je connais Jean $\rightarrow$ je sais que ceci et cela et cela...). Ces deux verbes commutent lorsque l'objet connaissance peut être résumé par une prédication unique (je sais la vérité) ou présenté comme 
“04_Bottineau_2” (Col. : RevueLangages) — 2016/7/2 — 12:56 — page 19 — \#19

Particularités du français calédonien et enjeux sémantiques, pragmatiques et cognitifs

un domaine glosable par un discours complexe irréductible à une déclaration unique (je connais la vérité). En calédonien, le remplacement systématique de savoir par connaitre revient à toujours mettre en scène la personne comme un sujet connaissant (détenteur d'un domaine épistémique correspondant à une chaîne discursive) plutôt que comme un sujet sachant (détenteur d'une proposition rapportable à une source énonciative singulière), même quand le fait connu est ponctuel :

(56) Hé ! tu connais, la trique elle est passée à ras les côtes à moi ! (source)

(57) Ben tu connais hier soir en regardant la télé j'ai eu l'idée de faire des bougnas en boittes de conserve (source)

Par rapport à tu sais que en français, dont le sens est 'tu es conscient que' ou 'apprends que', celui de tu connais que semble plus appuyé et sous-entendre 'tu auras remarqué que', 'tu n'es pas sans savoir que', 'tu peux bien t'imaginer que'. En contexte interrogatif, la polyphonie lexicale de connaître du côté du sujet connaissant convient idéalement pour l'interrogation relative, qui fait de la cible de l'interrogation un allocutaire lui-même polyphonique, concentrant plusieurs niveaux énonciatifs : Tu connais où que Dédé et son mari y zont été pêcher ? Employé interjectivement, Tu connais! exprime l'admiration.

\section{CONCLUSION}

L'observation du parler calédonien illustre comment la composition d'un parler issu de ressources hétérogènes peut engendrer un système de parole et une réinterprétation des composantes lexicales et grammaticales des systèmes de langue préexistants, dans le cadre d'une architecture cohérente, où des faits de langue divers semblent régis par des principes homogènes. La plupart des ressources grammaticales utilisées ne sont pas fondamentalement différentes de ce que l'on aurait dans d'autres zones de la francophonie, mais leur orchestration, cohérence, convergence et mise en scène par l'oralisation et dans le contexte idéologique et culturel calédonien " précipite » un mode de parole particulier qui, pris dans sa globalité, se distingue instantanément de tout autre. Ce système de parole est envisagé comme un code de la coordination intersubjective incarnée par la parole, et son utilisation permet en premier lieu la production d'états mentaux corrélatifs et conformes à des normes psychosociales caractéristiques d'une communauté et distinctives d'une « culture ». De ce fait, les " représentations » (de l'espace, du temps, des personnes, des activités) ne sont appréhendables qu'à travers les protocoles et routines des interactions verbales situées qui, en les convoquant, les constitue ; l'idée même de claquer un coup de langage 'parler sa langue vernaculaire' ne se comprend que dans l'univers interactionnel calédonien, et l'accès à cette idée - pour un extérieur comme l'auteur de cet article - passe inévitablement par un acte de simulation approfondie et régulée, laquelle est facilitée lorsque l'on peut comparer les processus observés à ceux d'autres parlers et dialectes munis de propriétés comparables, comme le picard. Cet appareil discursif de l'interaction, son aptitude à faire circuler et renouveler 


\section{Le français calédonien (Nouvelle-Calédonie), une variété régionale de français}

la normativité psychosociale et à naturaliser un mode d'existence culturel, fait du calédonien un objet d'intérêt général en linguistique générale et typologique, en particulier avec un regard enactiviste.

\section{CORPUS}

BERGER B (1985-2011), La Brousse en folie, Nouméa : Éditions La brousse en folie.

- tome 4: La marche des crabes mous dans le désert, 1989

- tome 10 : Petit Bateau mais gros la cale, 1996

- tome 13: Le mec sain malgré lui, 1999

- tome 14 : Le meilleur des Mandes, 2000

- tome 15 : Le jour le plus lent, 2001

\section{Références}

Auger J. (1994), Pronominal Clitics in Québec Colloquial French: a Morphological Analysis, PhD thesis, University of Pennsylvania.

Berrendonner A., Le Guern M. \& Puech G. (1983), Principes de grammaire polylectale, Lyon : Presses Universitaires de Lyon.

Bottineau D. (2012a), "Syntaxe et interlocution en français et en breton ", in C. Douay \& D. Roulland (éds), L'Interlocution comme paramètre, Rennes : Presses Universitaires de Rennes, 183-206.

Bottineau D. (2012b), "La fabrique de la langue, fabrique de l'humain ", in K. Nassikas, E. PrakDerrington \& C. Rossi (éds), Fabriques de la langue, Paris : Presses Universitaires de France, 161-197.

Bottineau D. (2012c), "La parole comme technique cognitive incarnée et sociale ", La Tribune Internationale des Langues Vivantes 52-53, 44-55.

BOTtINEAU D. (2014), "Explorer l'iconicité des signifiants lexicaux et grammaticaux en langue française dans une perspective contrastive (anglais, arabe) ", Le Français moderne 82 (2), 243-270.

CARRER P. et al. (1983)

CORNE C. (1989), "Un créole à base lexicale française en Nouvelle-Calédonie : le tayo ou le patois de Saint-Louis ", Études Créoles XII (2), 29-42.

DAROT M. (1998), "Les petits mineurs du caillou, le français et la mine en Nouvelle-Calédonie ", Le Français en Afrique 12, 105-116. [http://www.unice.fr/ILF-CNRS/ofcaf/12/Darot.htm]

DENEZ P. (1986), " Contraintes formelles de la structuration de la phrase bretonne ", in P. Carrer et al. (1986), Permanence de la langue bretonne : de la linguistique à la psychanalyse, Rennes : Institut Culturel de Bretagne, 19-44.

Douay C. \& RoulLAND D. (2012), "L'interlocution comme clé du contrastif ", in C. Douay \& D. Roulland (éds), L'Interlocution comme paramètre, Rennes : Presses Universitaires de Rennes, 77-94.

DouAy C. \& Roulland D. (2014), Théorie de la relation interlocutive : sens, signe, réplication, Limoges : Lambert-Lucas.

GUIRAUD P. (1967), Structures étymologiques du lexique français, Paris : Larousse. 
HILGERT E. (2012), "Nous autres / vous autres / eux autres, pronoms catégoriels ", in F. Neveu et al. (éds), Congrès Mondial de Linguistique Française - CMLF 2012, Paris : EDP Sciences, 1777-1792.

Pauleau C. (1995), Le Français de Nouvelle-Calédonie - Contribution à un inventaire des particularités lexicales, Paris : EDICEF-Association des Universités Partiellement ou Entièrement d'Expression Française [AUPELF].

Pauleau C. (2007), Mots de Nouvelle-Calédonie, éléments de recherche sociolinguistique sur le français calédonien: inventaire lexicographique polylectal, Nouméa: Centre de Documentation Pédagogique de Nouvelle-Calédonie (2 tomes).

WISSNER I. (2016b), "Le français des Isles: des Antilles à la Nouvelle-Calédonie ", Langages 203. (ce volume) 


\section{ABSTRACTS}

Didier Bottineau, The lexicon, morphology and syntax of neo-caledonian spoken French: semantic, pragmatic and cognitive aspects

Far from being anecdotic, the lexicon and morphosyntax of Caledonian spoken French display a whole range of coherent features which illustrate the way in which the psycho-social standards of verbal interactions in a human group bear upon usage and tend to profile the grammar of the language at the core of its system. With reference to the paradigm of enactive linguistics, this study shows how the routines of verbal interactions, driven by emotion and subjectivity, impose innovating and converging adjustments to heterogeneous linguistic facts.

Keywords : Caledonian spoken French, lexicon, morphosyntax, enunciation, verbal interactions, psycho-social standards, enaction

\section{RÉSUMÉS}

Didier Bottineau, Les particularités du français calédonien (lexique, morphosyntaxe) et leurs enjeux sémantiques, pragmatiques et cognitifs

Le français parlé calédonien présente un ensemble de caractéristiques cohérentes dans son lexique et sa morphosyntaxe qui n'ont rien d'anecdotique : elles illustrent la manière dont les modèles psychosociaux de l'interaction verbale dans une communauté constituée peut infléchir les usages au point de profiler la grammaire de leur langue au niveau même du système. En s'appuyant sur le paradigme de la linguistique enactive, cette étude montre comment une routine de l'interaction, mue par la subjectivité et l'émotion, impose à des faits de langue hétérogènes des ajustements innovants et convergents.

Mots-clés : français parlé calédonien, lexique, morphosyntaxe, énonciation, interactions verbales, émotions, modèles psycho-sociaux, enaction 